\title{
Perspectiva temporal futura: una aproximación al
} concepto

Future temporal perspective: an approximation to the

\author{
Paula Grasso Imig* \\ paula.grasso@uai.edu.ar \\ ORCID iD: \\ http://orcid.org/0000-0003- \\ 1977-6805
}

Universidad de Buenos

Aires*

\section{Para referenciar este artículo:}

Grasso, P. (2019).

Perspectiva temporal futura: una aproximación

al concepto. Revista

ConCiencia EPG, 4(1),

12-29. doi: https://doi.org/ 10.32654/CONCIENCIA

EPG.4-1.2

\section{Resumen}

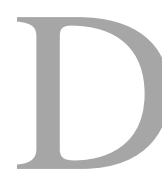

esde hace ya varias décadas que la perspectiva temporal futura se ha convertido en un importante objeto de estudio de la investigación psicológica. Sin embargo, resulta difícil encontrar un modelo que refiera de forma unificada su significado. El presente trabajo tiene como objetivo presentar un recorrido articulado acerca de los desarrollos más importantes sobre este constructo, para poder arribar a la definición más unificada hasta el momento. Partiendo de la idea que el procesamiento del tiempo es una función cognitiva, que permite organizar y dar coherencia a la vida de las personas, así como ha sido un factor primordial en la adaptación de la raza y que su asimilación despierta el interés en el hombre desde que existe la noción de tiempo, por ello, se pretende generar una revisión que permita a quienes investigan en la temática tener una idea general acerca de cómo se lo entiende y que a la vez, sirva para estructurar nuevos trabajos sobre el tema.

Palabras clave: perspectiva temporal, perspectiva temporal futura, dimensiones temporales. 
or several decades now, the future temporal perspective has become an important object of study of psychological research. However, it is difficult to find a model that refers in a unified way its meaning. The present work aims to present an articulated route about the most important developments on this construct, in order to arrive at the most unified definition until now. Starting from the idea that the processing of time is a cognitive function, which allows to organize and give coherence to the life of people, just as it has been a primordial factor in the adaptation of the race, and that its assimilation awakens the interest in man since there is the notion of time, it is intended to generate a review that allows those who research in the subject to have a general idea about how it is understood, and at the same time serve to structure new work on the subject.

Keywords: temporal perspective, future temporal perspective, temporal dimensions.

\section{Introducción}

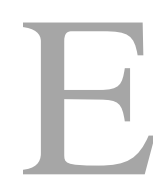

1 estudio del tiempo es una temática que ha ocupado buena parte de la investigación en el acontecer de la humanidad, habiendo varias disciplinas muy interesadas en ella, como desde hace ya algunos años también lo está la Psicología.

De forma más específica, éste se ha transformado en una parte relevante de la cognición humana y de cómo influye en la conciencia, pasando a ser objeto de estudio para esta disciplina hace ya más de un siglo (Díaz Morales, 2006a, 2006b).
En términos generales, la Perspectiva Temporal se define como la representación mental que las personas tienen acerca de diferentes acontecimientos (Zimbardo \& Boyd, 1999). En una aproximación a cómo será entendida en este trabajo, D'Alessio, Guarino, De Pascalis y Zimbardo (2003) la describieron como un proceso fundacional en el desempeño del sujeto, tanto como individuo como de éste en sociedad. Este proceso es no consciente y las categorías temporales involucradas cumplen un rol central en la relación que hay entre las experiencias de carácter personal y las de carácter social, lo que a su vez permite dar orden, coherencia y sentido a la vida del sujeto, ya sea en el pasado, presente o un posible futuro (D'Alessio et al., 2003; Sircova et al., 2014, 2015; Stolarski, Matthews, Postek, Zimbardo \& Bitner, 2013). Dicho de otra forma, se ha llegado a determinar que el tiempo les da sentido a las experiencias vividas, a la vez que permite autorregular el comportamiento presente y anticipar el futuro (Díaz Moralez, 2006a, 2006b; González Lomelí, Maytorena, Lohr Escalante \& Carreño Cruz, 2006).

\section{Perspectiva temporal: aspectos históricos y aproximación conceptual}

La discusión acerca del tiempo, así como la reflexión que este genera, tiene un lugar central en muchas disciplinas, entre las cuales se encuentran la Filosofía y la Psicología. En cualquiera de ellas puede observarse que implica tomar una postura sistemática respecto de la forma de existencia de la humanidad en el mundo, y también acerca de su devenir (Vázquez, Difabio de Anglat \& Noriega Biggio, 2016).

De esta manera, la Filosofía retoma la reflexión y se destacan dos ámbitos fundamentales para su debate: uno el de la Filosofía Natural, y otro el de la Antropología Existencial (Vázquez et al., 
2016), que son la base para las concepciones que luego desarrolla la Psicología.

En primera instancia, cabe destacar que los griegos ya habían realizado una aproximación a la forma de entender el tiempo y el acontecer de los hechos, utilizando dos conceptos que les permitieran describir y enmarcar distintos sucesos: cronos y kayrós. Respecto del primero, cronos, era la idea que les permitía realizar una medición del tiempo, poder evaluarlo; en esta instancia se incluyen, por ejemplo, las estaciones del año, los calendarios, y todo aquello que permitiera cuantificar los eventos, e incluirlos en algún grupo; en cuanto a kayrós es más específico y condicionado a la experiencia, ya que estaba asociado al momento en el que acontecen determinados sucesos, que son valorados como importantes por quienes participan de ellos; en este caso se consideran diferentes aspectos como los proyectos, los objetivos en torno a éstos y las expectativas que se manifiestan por estos hechos, y que se activan en la manera de actuar de cada persona; todo esto puede considerarse de carácter más cualitativo. No obstante, también puede destacarse un punto de índole cuantitativa a este concepto, ya que estos eventos tienen un principio y un fin (Galarraga, 2016; Savio, 2009).

Por otro lado, según Vázquez et al. (2016), pueden destacarse tres hitos que resultan fundamentales en la idea de comenzar a entender la percepción del tiempo, y son las concepciones de Aristóteles, San Agustín y Heidegger.

Así, en Aristóteles (1995) puede encontrarse la idea del tiempo asociada a las experiencias y a los cambios en las realidades naturales, que se suscitan a partir de dichas experiencias, en el sentido de movimiento según lo anterior y lo posterior; también, el autor explica que el tiempo tiene división tripartita, en la que cual el presente (o el ahora) representa la continuidad del tiempo, debido a que enlaza constantemente al pasado con el futuro, a la vez que se convierte en el límite del tiempo, porque mientras es el fin de uno es el comienzo del otro.

Para San Agustín (1946), quien describe al tiempo a partir de realizar una fenomenología de la experiencia, lo único que está es el momento de ahora, ya que "entre el ya no y el aún no, acaece como real únicamente el presente" (Vázquez et al., 2016, p. 3). Lo entiende como la constante de todo, porque es presente del pasado, presente del presente y presente del futuro, es decir, es la única instancia donde el sujeto puede experimentar la realidad. De esta forma, aparece como memoria cuando muestra lo que ha sucedido y lo hace presente, es visión cuando se vive lo presente como tal, y resulta expectación cuando surge la idea de lo que está por venir en la experiencia más íntima. En ese sentido, puede entenderse que no hay una representación del tiempo como movimiento, ya que, explicado de esta forma, y con un sentido estricto, el presente no alcanza extensión (Vázquez et al., 2016).

En su reflexión, San Agustín (1946) describe la idea del tiempo como distensión del alma, en la que ésta recuerda, atiende y espera según la experiencia. Esta idea sustenta el contexto para retomar la idea de lo que el autor entiende como tiempo trascendente, que no es tiempo en un sentido estricto (aunque toma un sentido coloquial cuando se relaciona con la experiencia interna), sino que aquella distensión ahora pasa a ser una intuición fija. A este tiempo lo denomina eternidad, y no es otra cosa más que un continuo presente, que no viene del pasado y tampoco huye hacia algún futuro.

Partiendo de una idea parecida se encuentra lo desarrollado por Heidegger (1924, 1962), para quien el presente, y todo 
lo que éste contiene, debiera llamarse tiempo auténtico. No obstante, aclara que no todo lo contenido en el presente es parte del momento actual, ya que éste guarda también lo que no está presente, como lo pasado o lo futuro.

En este contexto, otro aspecto que destaca el autor es que no todo lo presente pertenece al ahora, es decir, que no son lo mismo, sino que, de forma más específica y definitoria, hay que entender que el tiempo auténtico es tetradimensional, debido a que consta de la unidad de sus tres dimensiones; es justamente esto último lo que brinda al presente sus características.

Tener en cuenta esta breve reseña, de carácter filosófico, puede brindar un fundamento y una base a la idea que, por su parte, intenta desarrollar la Psicología respecto del tiempo y su forma de conceptualizarlo, así como de la influencia de sus dimensiones en la construcción de la identidad en las personas. La idea de finitud es una preocupación que acompaña al hombre desde que es tal en su idea de supervivencia. La noción más general permite entender que se trata de algo que no tiene principio ni fin, sin embargo, es una idea muy vaga, que no deja al hombre ordenar su existencia y es en esa idea, justamente, que lo divide para poder observar lo vivido, con la esperanza de especular lo que queda por vivir.

Entonces, puede resumirse que el tiempo y la forma de procesarlo es un tema central para el hombre y por eso, desde hace larga data se ha tornado en un tópico recurrente en diferentes áreas, entre las que se destacan la Filosofía, la Física, y desde hace más de un siglo la Psicología (Brenlla, Willis \& Germano, 2017). Esto último, debido al rol que el tiempo juega en la conciencia humana (Roeckelin, 2000); de hecho, para los psicólogos la percepción del tiempo es un elemento fundamental en la cognición, ya que, en términos generales, puede observarse que brinda sentido a las experiencias vividas, regula el comportamiento y permite anticipar el futuro (Díaz Morales, 2006; Fraisse, 1967; James, 1890; Lewin, 1942).

Se puede decir entonces, que la percepción del tiempo constituye una de las características básicas del comportamiento humano, y como indican Suddendorf y Corballis (1997), ha sido una variable psicológica determinante en el proceso de evolución de la especie humana ya que guía tanto el desarrollo como la conducta de las personas a partir de la planificación y establecimiento de objetivos.

Brenlla et al. (2017) consideran una diferenciación entre las concepciones del tiempo que se apoya justamente en las ideas de la Física, la Filosofía y la Psicología y que entienden como básica: el tiempo objetivo y el subjetivo. Así, en el caso del primero, se trata de un tiempo cronológico, y la Física lo entiende en términos de múltiplos o submúltiplos de segundos; en cuanto al tiempo subjetivo, que entra en el campo de exploración de la Filosofía y de la Psicología, hace referencia a la vivencia consciente sobre la duración y procesamiento de distintos momentos. De manera más específica, señalan que en el campo de la Psicología se han decantado dos grandes vertientes: una que sostiene la existencia de diferencias de carácter individual en cuanto a la perspectiva temporal, es decir, entiende que existen rasgos y otra, que se centra en el análisis de los fenómenos asociados a la percepción de duración.

Por su parte, Laplanche (1966) identifica a la dimensión del tiempo psicológico como el tiempo subjetivo (es decir el que marca la historia del propio sujeto), dentro del cual puede encontrase la perspectiva interna del tiempo, que es 
definida como la percepción subjetiva de cuán cerca se siente del pasado, el presente y el futuro (Vásquez Echeverria, 2011), así como también su captación y duración (Azzollini, González \& Gómez, 2004; Fraisse, 1984). En la misma línea, Thiébaut (1998) también la ha definido como el predominio con que el pasado, presente o futuro ocupan el espíritu del sujeto.

En el área de la Psicología, el concepto de perspectiva temporal fue introducido por Lewin (1935), quién lo comprende como una dimensión particular de la conducta humana, consistente en la visión que un individuo tiene, en un momento dado, de la totalidad de su futuro y su pasado. Según Lewin (1942), la perspectiva de tiempo referencia al pasado, presente y futuro cronológico, ya que ellos son parte de la vida psicológica del sujeto; postuló que la percepción del tiempo representa un elemento imprescindible en la cognición, dado que reviste de significación las experiencias que las personas viven a diario, regulando su comportamiento y anticipando el futuro. Según este autor, el presente de los sujetos se asocia a las experiencias vividas en el pasado, como también entiende que el presente estará en mayor o menor medida en aquello que se proyecta en el futuro y en las expectativas o metas que cada sujeto se propone alcanzar.

En suma, Nuttin (1985) considera que, la gestación de la perspectiva temporal depende de la elaboración cognitiva de la necesidad y del proceso consecuente de intenciones y proyectos.

En un sentido más elaborado, y ampliando lo anterior, Keought, Zimbardo y Boyd (1999) agregan que quienes tienen una orientación al presente poseen la habilidad para disfrutar el momento sin distraerse por las dificultades pasadas o las preocupaciones futuras; sin embargo, son incapaces de retrasar una gratificación y definir vías hacia metas razonables. Además, es posible que no presten atención a los avisos de que su conducta actual puede tener efectos negativos en el futuro. Aquellos con gran orientación al futuro, son buenos para establecer y lograr metas, además de plantear estrategias para cumplir obligaciones a largo plazo. De igual manera visualizan y formulan objetivos futuros, que influirán en decisiones y juicios en el presente.

En adición, Nuttin (1985) indica que el impacto de los eventos pasados y futuros sobre la conducta humana es tan importante que dichos eventos entran a formar parte del funcionamiento conductual presente, por lo menos a nivel cognitivo.

En resumen, siguiendo a Webster (2011), se puede definir a la perspectiva temporal como una dimensión estable, referida a las preferencias relativas que los individuos muestren hacia el pasado, el presente $\mathrm{o}$ al futuro, en función de los aspectos cognitivos, actitudinales y comportamentales. Algunos autores, como Kruger, Reischl y Zimmerman (2008), proponen que es, también, un mecanismo psicológico que permite el desarrollo funcional y la adaptación de los seres humanos.

\section{Perspectiva temporal futura (PTF)}

En lo que refiere específicamente a la perspectiva temporal futura (PTF), se encuentran varias definiciones. En cuanto a los orígenes del constructo, pueden observarse los aportes de Bleger (1963), sobre la acción recíproca que explica la manera en que experiencias del pasado se asocian a la forma como las personas viven el presente y en cómo esto repercute en la proyección del futuro. Asimismo, explica que el futuro condiciona la forma en que se vive el presente y la valoración respecto del pasado, de la propia historia. Sumando lo 
desarrollado por Lewin (1942), la PTF es el grado y la forma en que el futuro cronológico se integra en el espacio de la vida del individuo.

Por otra parte, en lugares como Europa, la conceptualización de la PTF tiene una larga historia en la Psicología. En ese sentido, se observan varios autores que han esbozado diferentes ideas. Así, Lersch (1966) expresa que tanto pasado como futuro se encuentran contenidos en la vivencia presente; de esa manera, todo presente es a la vez un adelanto del futuro. Esta apreciación articula la vida anímica del sujeto con la orientación en la que éste se encuentre y lo disponen a la realización de un estado que aún no existe.

Para Fraisse (1963), el futuro tendrá lugar siempre y cuando éste se perciba como posible de ser realizado; este futuro es movido por un determinado deseo previo, que es el motor que pone en marcha las estrategias necesarias para afrontar dicho futuro. De esta forma, la postura de este autor se resume en la idea de que tanto la necesidad de un deseo por algo, como la conciencia de que puede ser logrado, deben estar presentes para que haya futuro.

En una línea parecida, Nuttin (1964) va a indicar una asociación entre el futuro y la motivación, es decir, no solamente lo va a entender como una consecuencia y aprendizaje de los eventos pasados, sino que, le va a dar al futuro una cualidad de metaobjeto. De esta manera, el futuro se transforma en un espacio motivacional de primera categoría. No obstante, esta cualidad se limita a una orientación general, a un futuro inmediato, debido a que su impulso es la necesidad. Luego, la aparición de perspectivas profundas se da gracias al desarrollo de las funciones cognitivas superiores y a su influencia en la elaboración de las necesidades, sobre todo en aquellos procesos de establecimiento de metas. Es así que la anticipación se va desligando gradualmente de la situación, para formar parte de un proceso superior. Entonces, de manera general, Nuttin (1985), entiende que la perspectiva temporal es la representación mental que los seres humanos realizan acerca de diferentes acontecimientos, haciendo referencia tanto al pasado como al futuro, pero, es la elaboración de proyectos la que da origen a la orientación futura.

En adición a estas ideas, para Gjesme (1981) la orientación del tiempo futuro de un individuo se desarrolla de forma gradual, hasta convertirse en una característica relativamente estable de la personalidad, en términos de la capacidad general para anticipar e iluminar el futuro, incluyendo la elaboración cognitiva de los planes y proyectos, y reflejando el grado de preocupación y compromiso en el futuro.

De una manera más amplia, Nurmi (1991), caracteriza la PTF como un proceso biológico complejo, que se constituye a partir de las expectativas que las personas tienen de sí misma en el futuro y el grado en que se reflexiona acerca de ello. La PTF se encuentra asociada al contexto social y cultural del sujeto, así como también a la etapa del desarrollo en que se ubica.

Por su parte, Husman y Lens (1999) expresan que la PTF es el grado y la manera en la cual el futuro cronológico es integrado en el espacio vital presente del individuo, a través, de procesos motivacionales de establecimiento de metas. Zimbardo y Boyd (1999), asocian la PTF a la capacidad de entender las consecuencias de los actos a realizar, a tener objetivos claros, poder tolerar tanto el placer como las tensiones que puedan aparecer a partir del proceso de llevar a cabo las metas propuestas. A fin de integrar todo lo explicado, puede decirse que la perspectiva temporal futura es entendida como las creencias o la orientación de un sujeto en torno al futuro, a partir de 
diferentes objetivos que son distantes temporalmente; de manera usual se la asocia a una representación que está relacionada con diferentes aspectos de la vida. En ese sentido es posible entenderla como una actitud en relación con un objetivo específico, en un tiempo determinado.

\section{Enfoques predominantes sobre la PTF}

\section{Perspectiva según Nuttin.}

Para Nuttin (1985), la perspectiva temporal futura es el constructo que propone una configuración de objetos temporales futuros asociados a la cognición de los sujetos, que a la vez se integran en el presente, de manera que influyen en este mismo. Una característica fundante es la motivación, ya que es ésta, la que genera las representaciones en el presente y que luego impactan en la conducta. En ese sentido, la PTF contempla diferentes aspectos, a saber:

i) La extensión que tienen las metas, es decir, cómo se ubican éstas en relación con un futuro cercano, lejano, o a un presente abierto;

ii) La cantidad de un tipo de metas que se ubica en cada uno de esos períodos; y

iii) La especificidad que cada una de estas metas posee para el sujeto que las desarrolla; esto último es central, ya que da cuenta del correlato entre la realidad y el cumplimiento de dichas metas (Lens, 1998; Madueño Ruiz, 2017; Nuttin, 1985).

Por otra parte, una observación interesante acerca de la manera que tiene este autor de entender este constructo la realiza Galarraga (2016), cuando señala el origen del término, y lo diferencia en cuanto las nociones espaciales, por una parte, $y$ temporales, por otro. En ese sentido, Nuttin (1985) sostiene que el término perspectiva tiene un carácter espacial, cuya conceptualización hace referencia a distancias medidas objetivamente. En cuanto a la noción de temporalidad, la particularidad está puesta en que los análisis se realizan en función de los intervalos de tiempo; el autor también resalta que no prestar atención a esto puede generar algún tipo de confusión respecto de esta terminología. De esta manera, entender la diferencia entre estas dos formas de referir sobre la perspectiva, permite señalar que la perspectiva temporal describe la representación mental que las personas tienen acerca de determinados sucesos y no procesos de percepción (Galarraga, 2016; Nuttin, 1985).

Además, el autor operacionaliza el constructo a partir de dos dimensiones: una cognitiva, que alude a la representación, y otra dinámica, que hace referencia al valor motivacional de las metas distantes (González \& Daura, 2012).

En el caso de la primera dimensión se asocia a la capacidad del sujeto al momento de ubicar los proyectos o las metas en el futuro, ya sea cercano o lejano; para algunos autores (Husman, 2001) esto se relaciona con la instrumentalidad de dichos proyectos o metas, es decir, que tiene que ver con la valoración que se realiza de estas mismas en un momento próximo para después alcanzar las que se encuentran más distantes, mientras que Nuttin (1982) lo va a llamar directamente motivación instrumental, ya que esto toma un rol central en el momento que se forman los proyectos y sus fines. En ese sentido, González y Daura (2012) explican, que el grado de motivación que el individuo tenga al momento de la creación de proyectos será el que estimule y dirija la actividad cognitiva, así como la manipulación del contexto, creando -a partir de la necesidad- conductas que permitan abrir el camino hacia los objetos satisfactorios, lo que formará la red que 
estructure el plan de acción para el proyecto. Lo interesante de este punto es que a medida que el acto se interpreta como parte funcional de esa red, se inviste de una significación, que se transforma en el camino conductual hacia el fin.

En lo que refiere a la dimensión dinámica lo entiende como la valoración que se le imprime a los acontecimientos futuros, siendo aún más precisos se refiere a la valoración de las metas distantes; así, puede entenderse las conductas de las personas son intencionales en la medida que se encuentre orientada a alcanzar las metas establecidas. A partir de esto, se entiende que existen, de manera subyacente, ciertos procesos motivacionales que determinan en buena medida los comportamientos (González \& Daura, 2012; Nuttin, 1980).

En función de lo anterior, González y Daura (2012) explican que la PTF, por lo tanto, presupone que el presente contenga la representación mental del futuro, así como también debe comprender las metas motivacionales y la ubicación temporal de estas últimas. Son justamente estas metas motivacionales (u objetos motivacionales) lo que van a conformar el contenido de la PTF, así como su ubicación temporal aquello que defina si el tiempo en que se concreten será en corto, medio o largo plazo.

Otro aspecto para resaltar en el desarrollo de este autor es la diferenciación que propone entre perspectiva temporal, actitudes temporales y orientación temporal (Galarraga, 2016). Con respecto a las actitudes temporales (que son los aspectos negativos, neutros o positivos con los cuales se valora a cada uno de los tiempos pasado, presente $\mathrm{y}$ futuro) se las considera importantes de manera especial, debido a que producen un gran impacto en la motivación, por lo tanto, en buena medida determinan la forma en la que los sujetos asumen su pasado, viven el presente y se aproximan al futuro (González \& Daura, 2012; Nuttin, 1980). En ese sentido, Zaleski (1994) explica que la actitud que se toma hacia el futuro puede acompañarse tanto de temores y/o ansiedad como de esperanza, esto debido a las experiencias vividas y a la forma en la fueron procesadas y elaboradas. No obstante, también señala que, si bien es probable que una mala experiencia pasada, tenga consecuencias en el presente, no necesariamente la actitud hacia el futuro debe ser negativa, en ocasiones estas valoraciones pueden invertirse.

En resumen, puede decirse que el desarrollo de la PTF se asocia, al tiempo que depende de la forma cognitiva en que se elabore una determinada necesidad, así como del desarrollo consecuente de los proyectos e intenciones (Nuttin, 1985; Vázquez et al., 2016). Así, la forma de anticipar el futuro se relaciona a las experiencias pasadas y son éstas las que se integran al presente y determinan la proyección (Madueño Ruiz, 2017).

\section{Desarrollo de Nurmi}

Otro de los autores que propone un enfoque de relevancia en cuanto al desarrollo conceptual de la PTF es Nurmi. En ese sentido, Crespi (2010) señala que para este autor, tanto los eventos como los resultados futuros conforman un aspecto central respecto de los pensamientos y los comportamientos en las personas. En esa línea, una posible definición de la PTF puede hacer referencia a aquellas expectativas que las personas tienen de sí mismas en el futuro, a la vez que incluye el grado de reflexión acerca de ellas (Nurmi, 1991).

Un aspecto que se destaca en el desarrollo de este autor, que es a la vez un gran complemento a su idea, es la importancia que le brinda al contexto, así como a las características socio - culturales que éste provee a cada individuo por el solo 
hecho de pertenecer a él (Galarraga, 2016; Nurmi, 1991); en ese sentido, también suma la importancia de las diferentes etapas del desarrollo, entendiendo a la interacción entre el primero y estas últimas como necesarios e inseparables (Nurmi, 1991, 1994). Respecto a esto, Galarraga (2016) explica que, para este autor, los diferentes momentos del ciclo vital que atraviesa el sujeto implica actuar en función de ciertos roles o tareas, que irán variando, dependiendo del momento del desarrollo en que se encuentre.

De esta manera, Nurmi (1991) entiende que aquellos roles son los que conformarán las bases sobre las cuales se producirán las metas o proyectos, así como la forma de alcanzarlos.

En resumen, puede entenderse que para este autor la PTF es un constructo tanto de carácter biológico, como social y cultural, que implica tener en cuenta los roles que esto le ha ido suponiendo al sujeto, así como las metas motivacionales que a partir de esto se genera. Al mismo tiempo, señala a la PTF como multidimensional, constituido por tres componentes centrales: la motivación, la planificación y la evaluación prospectiva (Crespi, 2010; Galarraga, 2016; Nurmi, 1991). En lo que refiere al componente motivacional, tiene que ver con la extensión del tiempo hacia el futuro, lo que implica tener en cuenta la menor o mayor distancia temporal hacia dicho futuro y las metas, objetivos o intereses que los componen, debido a que es un elemento de la conducta motivada (Crespi, 2010; Díaz Morales, 2006). Galarraga (2016) agrega que también, que esta conducta, implica anticipar el futuro y construir metas, que luego son integradas y organizadas en un sistema jerárquico, dependiendo del grado madurativo de la persona.

En cuanto a la planificación, el segundo de los componentes mencionados, Crespi (2010) sostiene que se asocia a los conocimientos y a la información que se tiene respecto de las metas establecidas, así como del contexto futuro; además, tiene en cuenta la complejidad de los proyectos elaborados y en qué grado se van realizando en la realidad, es decir, que puede obtenerse información acerca del estado en que se encuentra la planificación al momento presente (Crespi, 2010; Crespi \& Mikulic, 2011; Nurmi, 1991). Por último, Galarraga (2016) señala que este componente, a su vez, se divide en tres momentos: "representación de metas, planificación de metas y la puesta en marcha de una acción para alcanzar las metas" (p. 50).

Respecto del tercer y último componente, la evaluación prospectiva, se entiende como a la factibilidad de aquellas metas establecidas, tanto como de los planes que se lleven a cabo para alcanzarlas, teniendo en cuenta la situación presente, así como también las competencias y habilidades con las que cada individuo cuenta para llevarlas a cabo (Crespi, 2010; Díaz Morales, 2006).

\section{Explicación de Lens}

Lo desarrollado por este autor sigue la línea de lo explicado anteriormente con Nuttin. En primera instancia, para contextualizar, realiza una aproximación sobre la definición de perspectiva temporal retomando a Lewin (1942) y va a decir que se trata de un concepto que refiere al pasado, presente y futuro debido a que son instancias que se conforman en el espacio de la vida psicológica de las personas; de esta manera, se desprende que la PTF, como consecuencia, será la forma y el grado con el cual el futuro cronológico se integre a la vida del sujeto. En ese sentido, entiende que existe una analogía entre la integración del futuro al presente con la integración de lugares que son más o menos remotos al espacio de la vida actual (Lens, 1998). 
Para este autor, la conciencia acerca del lugar, así como de las distancias entre los momentos, o los intervalos en el tiempo (ya sea pasado o futuro), son necesarios, aun cuando no resulten suficientes al momento de darles un efecto psicológico. Explica que la distancia psicológica de un lugar específico puede variar de forma considerable entre personas que habitan un mismo lugar; así, de la misma manera que existe la apreciación psicológica acerca del mundo espacial, "existen varias y profundas diferencias en la extensión o profundidad de la perspectiva de tiempo futura" (Lens, 1998, p. 70)

Además, establece una diferencia entre aquellas personas que tienen una corta y una larga PTF; en el caso de las primeras hace referencias a quienes solo incluyen en su vida temporal el futuro cronológico inmediato, estos sujetos no toman en cuenta en el tiempo presente lo que pudiera aparecer después de esa inmediatez contemplada. En el caso de las segundas, su vida temporal presente contempla el futuro lejano, es decir que no tienen problemas en integrar a dicho presente aquellos aspectos relacionados a las acciones o eventos que los motivan para tal futuro. En ese sentido, la PTF es conceptualizada como aquellas características que adquiere la personalidad, y que resulta tanto de las metas motivacionales como de la demora en la gratificación (Lens, 1986, 1998; Nuttin y Lens, 1985).

En función de lo explicado hasta el momento, cabe destacar que el carácter motivacional resulta central en lo desarrollado por este autor. De esta manera, describe que, en un nivel superficial, las personas elaboran de forma cognitiva $y$ luego concretizan, tanto sus motivos como sus deseos a partir de la elaboración tanto de metas motivacionales específicas como de estructuras medios-fin o proyectos motivacionales (Gjiesme, 1982; Herrera,
Matos, Martínez \& Lens, 2015; Lens, 1998). Dichas metas motivacionales, así como también los planes conductuales, se pueden caracterizar por sus contenidos o por el dominio motivacional al que pertenezcan (como por ejemplo, curiosidad, poder, éxito, alimentación) y por su ubicación témporoespacial.

Para contextualizar aún mejor lo explicado, Lens (1998) señala que, por definición, las metas motivacionales tienen de forma anticipada un carácter positivo o negativo y que, de manera casi obvia, están ubicadas en el futuro. Sin embargo, la distancia temporal hacia las sub-metas y/o hacia las metas finales puede variar desde corta hasta larga, dependiendo del contenido de las mismas. El hecho de formular estas metas motivacionales a la distancia, así como de generar los proyectos que permitan alcanzarlas, creará una perspectiva a futuro de largo plazo (o extendida). A partir de esto último, brinda un aspecto nuevo, que se suma a la definición entendiendo que la PTF también, "puede ser definida como la anticipación presente de las metas futuras" (Lens, 1998, p. 70).

Por otro lado, si se compara a quienes tienen una PTF extendida con aquellos quienes tienen una PTF corta puede observarse que, en el caso de las primeras, poseen muchas más metas a largo que a corto plazo. Como consecuencia de esto, Nuttin y Lens (1985) sostienen que la perspectiva de tiempo futuro puede evolucionar a partir de establecer estas metas motivacionales, que están conformadas por la relación distante de los objetos-meta que procesan las personas.

Por último, además de lo referido, este autor (al igual que Nuttin) también contempla los aspectos cognitivo y dinámico de la PTF como una característica de la personalidad. Así, en cuanto al aspecto cognitivo explica que puede entenderse como la disposición que tiene el individuo 
para anticipar en el momento presente tanto los efectos inmediatos de las acciones potenciales, como las consecuencias esperadas de estas mismas. En cambio, el aspecto dinámico se entiende en función de que el individuo tiene la disposición de atribuir un valor alto a las metas, incluso cuando éstas son proyectadas a un futuro no tan cercano (Herrera et al., 2015; Lens, 1998).

\section{Enfoque de Thiébaut}

Basado en el desarrollo de Nuttin, por un lado (González \& Daura, 2012), y en el de Nurmi por otro (Galarraga, 2016), Thiébaut (1998) entiende acerca de la perspectiva temporal que se trata de un proceso que permite al individuo prever y anticipar acontecimientos del futuro, que no son parte de los contenidos en el presente; sobre éstos, Galarraga (2016) aclara que pueden ser "reales, posibles, imposibles o imaginarios" (p. 50).

Al mismo tiempo, Thiébaut (1998) la entiende como el predominio que el presente, el pasado o el futuro establece en el individuo; a partir de esto se espera que los pensamientos de una persona hacia alguno de aquellos tiempos (pasado, presente o futuro) influya de forma dinámica sobre la experiencia del sujeto, así como sobre la motivación y diferentes aspectos del comportamiento (Boniwell, 2009; Thiébaut, 1998; Vázquez et al., 2016).

De manera más específica, la perspectiva temporal futura representa la acción que es ejercida al momento de producir las intenciones comportamentales (Galarraga, 2016). Al igual que los anteriores autores, éste también destaca diversas dimensiones para este constructo: la orientación temporal, que trata del predominio relativo que se tenga acerca del pasado, presente o futuro; la densidad, que refiere a la riqueza de los contenidos de cada una de las etapas temporales; la extensión, que describe la longitud en el tiempo de meta a alcanzar; y la coherencia o realismo, que en sí misma tiene diferentes matices, y que indica, por una parte, la probabilidad que tienen las metas propuestas de ser realizadas en el futuro y, por otra, la existencia de nexos entre estas metas ubicadas a lo largo del tiempo; también, implica tener en cuenta la gradación entre el carácter más real y el más ilusorio que posean cada una de estas metas (González \& Daura, 2012; Thiébaut, 1998).

En suma, Galarraga (2016) describe la afectividad, que hace referencia al monto afectivo que se da de forma expresa al futuro; la precisión, que indica el grado de claridad con el cual son afrontadas las metas o proyectos; y la continuidad en el tiempo, que determina la forma en la que cada persona organiza dichas metas o proyectos.

González y Daura (2012) advierten que las distintas dimensiones tienen dinámicas independientes, no necesariamente deben estar relacionadas unas o con otras o darse en simultáneo.

\section{Modelo de PTF de Zimbardo y Boyd}

Para Zimbardo y Boyd (1999) la PTF debe ser entendida en términos de orientación temporal, lo que implica reconocer la tendencia que el sujeto tiene de estar centrado en el pasado, en el presente o el futuro, dicho en otras palabras, se trata de la prevalencia de una de éstas sobre las otras. Es un proceso que se encuentra en el origen del comportamiento, que permite a las personas organizar su conducta en diferentes momentos, brindando sentido y coherencia a la experiencia, lo que al mismo tiempo brinda orden tanto a los sucesos como a la vida misma. A pesar de no ser tangible del todo, se trata de un proceso que tiene lugar en las personas, y que permite organizar tanto las experiencias de carácter individual como social. 
Una característica importante de los marcos temporales es que contribuyen a la codificación, almacenamiento y rememoración de situaciones que han sido experimentadas, así como también ejercen un rol primordial para la construcción de nuevas metas, expectativas y objetivos futuros. De esta manera, se observa que se trata de una dinámica constante, que se retroalimenta.

A pesar de todo esto, Zimbardo y Boyd (1999) resaltan que, en la mayoría de los casos, se trata de un proceso no consciente, es decir que aun cuando quedan adscriptas las experiencias más personales o sociales en alguno de estos marcos 0 categorías temporales, que contribuyen a la asignación tanto de orden como de significado y coherencia a lo vivido, las personas no siempre saben que lo están haciendo o proveen de intensión a la acción.

Para Vázquez et al. (2016) es importante hacer notar el peso relativo que cada uno de estos marcos temporales adquiera para el sujeto, ya que a esto establece diferencias importantes respecto de las decisiones y comportamientos que se tienen; por esto resulta importante analizar las modalidades que cada una de estas categorías temporales adquiera en cada uno.

Respecto de las preferencias por alguna de las dimensiones temporales, Zimbardo, Keough y Boyd (1997) señalaron que las mismas pudieran funcionar como un rasgo estático de la personalidad, lo que también tiene algunos costos, y que para Boniwell (2009) en ocasiones pudieran ser desadaptativos. Cabe aclarar que esto último produjo el desarrollo de un concepto integrador, que es la perspectiva temporal balanceada, que combina al pasado, presente y futuro en una dinámica flexible, que le permite al sujeto responder a las exigencias del momento de forma armoniosa (Boniwell \& Zimbardo, 2004; Zimbardo \& Boyd,
2005; Vázquez et al., 2016; Zimbardo, 2002).

$\mathrm{Al}$ igual que los autores presentados en los enfoques anteriores, Zimbardo y Boyd (1999, 2008) describen diferentes dimensiones para la PTF, en este caso se trata de cinco formas en cuales se le da sentido al tiempo, se lo valora, y que se conforman en base a las tres categorías importantes ya conocidas: pasado, presente y futuro. Galarraga (2016) explica que esto permite dar cuenta de la forma en que las personas se refieren al propio tiempo. Las cinco dimensiones propuestas por Zimbardo y Boyd (1999) son las siguientes:

- Pasado positivo: se asocia a las experiencias que tuvieron las personas y que recuerdan como agradables y placenteras.

- Pasado negativo: refiere a los sucesos que tienen carga negativa, ya que resultaron difíciles $\mathrm{y}$ complicados.

- Presente hedonista: tiene que ver con la búsqueda de sensaciones que producen placer en el marco de las situaciones que se viven de forma diaria.

- Presente fatalista: se relaciona con las experiencias actuales de las personas, pero que generan ansiedad y miedo.

- Futuro: se encuentra referido a planifica las acciones en función de los objetivos que se establecieron para alcanzar determinadas metas.

La dinámica de estas categorías muestra que quienes tienen una orientación temporal enmarcada hacia el presente están más pendientes de lo percibido en un determinado momento; entre las características observadas puede notarse que son de pensamientos concretos, y que -en general- no se los nota interesados en 
acontecimientos pasados o venideros, así como tampoco están al pendiente de las consecuencias de sus actos. En cambio, quienes presentan una orientación hacia el pasado si bien tampoco demuestran interés en los sucesos del futuro, sí mantienen una conducta más estable a lo largo del tiempo, y se enfocan en los compromisos que asumen, siendo estos de la índole que sea (académica, familiar, laboral). Para quienes muestran una orientación hacia el futuro dan cuenta de un interés asociado a esta orientación temporal, así como en las consecuencias de dichos actos; en general poseen objetivos claros, lo que les ayuda a mantener el equilibrio entre el placer, la tolerancia y las tensiones entre las distintas situaciones que se produzcan al momento de querer llevar a cabo las metas que se proponen (Galarraga, 2016; Zimbardo \& Boyd, 1999).

Para resumir lo explicado sobre este modelo, que a su vez encuadra la mayoría de los desarrollos realizados sobre esta temática, puede decirse que los autores entienden que la perspectiva temporal es un proceso que se encuentra en la génesis del comportamiento humano, tanto a nivel individual como social; se trata de un proceso no consciente, pero que igualmente permite realizar análisis, así como también organizar, el continuo devenir de la conducta en distintos marcos temporales, y esto permite que los comportamientos se doten de sentido y coherencia (Brenlla et al., 2017; Zimbardo \& Boyd, 1999).

Estos marcos temporales, en los cuales las personas ubican sus experiencias, representan la forma en la que se dividen las experiencias en cuanto al devenir de la propia existencia, aunque se esto se realice de manera automática y no consciente. Ello supone una articulación balanceada entre las distintas instancias temporales, lo que lleva al sujeto a tener flexibilidad y armonía conductual cuando debe enfrentar demandas situacionales, y cuando se trata del logro de una competencia temporal para lograr una mejor adaptación (Brenlla et al., 2017; Zimbardo \& Boyd, 1999; Zaleski, 1994). No obstante, dicho equilibrio puede verse alterado cuando alguna de estas instancias temporales registra un predominio sobre el resto, lo que promueve un sesgo en la perspectiva temporal del individuo; en ese sentido, los autores consideran la idea de que la perspectiva temporal conforma un estilo disposicional relativamente estable, y por este motivo es también considerada como rasgo de la personalidad (Boniwell \& Zimbardo, 2004; Boyd \& Zimbardo, 2005; Brenlla et al., 2017; Vázquez et al., 2016; Zimbardo et al., 1997; Zimbardo, 2002; Zimbardo \& Boyd, 1999).

Tabla 1

Desarrollos relevantes sobre la PTF

\begin{tabular}{llll}
\hline Autor / es & Año & Definición & Dimensiones \\
\hline Nuttin & 1985 & $\begin{array}{l}\text { Configuración de objetos temporales } \\
\text { futuros asociados a la cognición de los }\end{array}$ & - Cognitiva \\
& & & \\
& & sujetos, que a la vez se integran en el \\
& presente, de manera que influyen en este \\
& mismo. \\
& \\
& Una característica fundante es la \\
& motivación.
\end{tabular}




\begin{tabular}{|c|c|c|c|}
\hline Nurmi & 1991 & $\begin{array}{l}\text { Aquellas expectativas que las personas } \\
\text { tienen de sí mismas en el futuro, a la vez } \\
\text { que incluye el grado de reflexión acerca de } \\
\text { ellas. }\end{array}$ & $\begin{array}{l}\text { - } \text { Motivación } \\
\text { - } \text { Planificación } \\
\text { Evaluación } \\
\text { prospectiva }\end{array}$ \\
\hline Lens & 1998 & $\begin{array}{l}\text { La forma y el grado con el cual el futuro } \\
\text { cronológico se integre a la vida del sujeto. }\end{array}$ & $\begin{array}{l}\text { - Cognitiva } \\
\text { - } \quad \text { Dinámica }\end{array}$ \\
\hline Thiébaut & 1998 & $\begin{array}{l}\text { Se trata de un proceso que permite al } \\
\text { individuo prever y anticipar } \\
\text { acontecimientos del futuro, que no son } \\
\text { parte de los contenidos en el presente. }\end{array}$ & $\begin{array}{ll}\text { - } & \text { Orientación } \\
\text { temporal } \\
\text { - } & \text { Densidad } \\
\text { - } & \text { Coherencia o } \\
& \text { Realismo }\end{array}$ \\
\hline $\begin{array}{l}\text { Zimbardo } \\
\& \text { Boyd }\end{array}$ & 1999 & $\begin{array}{l}\text { Es un proceso que se encuentra en la } \\
\text { génesis del comportamiento humano, tanto } \\
\text { a nivel individual como social; es no } \\
\text { consciente, pero que igualmente permite } \\
\text { realizar análisis, así como también } \\
\text { organizar, el continuo devenir de la } \\
\text { conducta en distintos marcos temporales }\end{array}$ & $\begin{array}{l}\text { - Pasado positivo } \\
\text { - Pasado negativo } \\
\text { - Presente } \\
\text { Hedonista } \\
\text { - Presente } \\
\text { fatalista } \\
\text { - Futuro }\end{array}$ \\
\hline
\end{tabular}

\section{Conclusión}

A partir de lo desarrollado puede observarse que el estudio del tiempo genera gran interés en varias disciplinas; quienes se dedican al estudio de éste constructo, reconocen en esto, una trayectoria que data de mucho tiempo, aunque en el área de la Psicología su tradición es de poco más de un siglo. Asimismo, cada autor ha buscado dar una idea integrada, que permitiese entender al tiempo y sus categorías y la forma de integrar esto en la vida de cada individuo. Pero, al igual que con la mayoría de las variables psicológicas, el límite ha quedado en cada forma de interpretar el constructo, así, más allá de la integración amplia que algunos autores han realizado (como Zimbardo y Boyd), todavía no hay un consenso para definirlo de forma genérica. Como puede verse reflejado a lo largo de este trabajo, esto podría deberse también, a que en estas diferentes posturas desarrolladas a través de los años- las perspectivas elaboradas responden tanto a diferentes criterios, como definiciones y categorías, las cuales no llegan a quedar delimitadas dentro de una idea unificadora. Queda claro que describe lo necesario acerca de las características de la perspectiva temporal futura, contemplando aspectos individuales y sociales, así como patológicos, pero hay que tener en cuenta que esto es aún muy general y que quedan muchos aspectos para revisar.

Hay que tener en cuenta que estas ideas que no llegan a agotar la noción que se tiene del tiempo y sus consecuencias es también lo que hace de esta variable un objetivo interesante de seguir estudiando, pues se trata de una característica que atraviesa a todos de manera inherente; es decir, es casi imposible despojarse de la idea de orden en la vida cotidiana (contemplando desde lo más simple a lo más complejo) sin especular acerca de cómo será organizado el tiempo que se tiene. En ese sentido, tener una medida que permita asociar las características del tiempo y su devenir al 
accionar de las personas, así como la construcción de identidades resulta de gran relevancia.

Por último, hay que dejar claro que este trabajo no contempla todos los desarrollos acerca de esta variable, por lo tanto, no logra ser exhaustivo. No obstante, trata de mostrar una línea que se encamine hacia una idea unificada de entender este constructo, buscando un punto de cohesión entre diferentes desarrollos. Se espera poder profundizar en estos aspectos, de manera de poder lograr una idea totalizadora acerca de cómo entenderlo, así como a sus diferentes componentes.

\section{Referencias}

Aristóteles. (1995). Física. Madrid: Gredos.

Azzollini, C., González, F. \& Gómez, M. (2004). La relación entre la capacidad de atención dividida y la estimación prospectiva del tiempo. Interdisciplinaria, 21(2), 195-212.

Bleger, J. (1963) Psicología de la conducta. Buenos Aires: Paidós.

Boniwell, I. (2009). Perspectives on time. En C.R. Snyder \& S.J. Lopez (Eds.), The Oxford Handbook of Positive Psychology (2da. ed., pp. 295-302). New York: Oxford University Press.

Boniwell, I. \& Zimbardo, P. (2004). Balancing one's time perspective in pursuit of optimal functioning. En P.A. Linley \& S. Joseph (Eds.), Positive psychology in practice (Cap. X, pp. 165-178). Hoboken, NJ: Wiley.

Boyd, J.N. \& Zimbardo, P.G. (2005). Time perspective, health, and risk taking. En A. Strahman \& J. Joireman (Eds.). Understanding behavior in the context of time: Theory, research and applications (pp. 85-107). Mahwah, NJ: Erlbaum.

Brenlla, M. E., Willis, B. \& Germano, G. (2017). Estimación del tiempo y perspectiva temporal en etapas de la adultez. Investigaciones en Psicología, 21(1), 27-34.
Crespi, M. (2010). Estudio de la perspectiva temporal futura en una muestra de liberados condicionales. II Congreso Internacional de Investigación $y$ Práctica Profesional en Psicología XVII. Jornadas de Investigación Sexto Encuentro de Investigadores en Psicología del MERCOSUR. Facultad de Psicología - Universidad de Buenos Aires, Buenos Aires, 2010.

Crespi, M. \& Mikulic, I.M. (2011). Reinserción social: estudio de la perspectiva temporal futura en sujetos que han recobrado su libertad. Anuario de Investigaciones, 18(1), 401-408.

D’Alessio, M., Guarino, A., De Pascalis, V. \& Zimbardo, P. G. (2003). Testing Zimbardo's Stanford Time Perspective Inventory (STPI) - Short Form. An Italian study. Time \& Society, 12(2/3), 333-347.

Díaz Morales, J. F. (2006a). Estructura factorial y fiabilidad del Inventario de perspectiva Temporal de Zimbardo. Psicothema, 18 (3), 565-571.

Díaz Morales, J.F. (2006b). Perspectiva temporal futra y ciclo vital. Anales de Psicología, 22 (1), 52-59.

Fraisse, P. (1963). The psychology of time. Westport: Greenwood. 
Fraisse, P. (1967). Psychologie du temps. Paris: PUF

Fraisse, P. (1984). Perception and estimation of time. Annual Review of Psychology, 35, 1-36.

Heidegger, M. (1999). El concepto de tiempo. Madrid: Trotta. Trabajo original publicado, 1924.

Heidegger, M. (2009). Tiempo y ser. Madrid: Tecnos.

Galarraga, M. L. (2016). Perspectiva temporal futura, apoyo social y afrontamiento al estrés en alumnos de escuelas secundarias de la Provincia de Buenos Aires (Tesis de Maestría en Psicodiagnóstico y Evaluación Psicológica). Universidad de Buenos Aires, Buenos Aires. Argentina.

Gjesme, T. (1981). Is there any future in achievement motivation? Motivation and Emotion, 2, 115-138.

Gjiesme, T. (1982). Psychological goal distance: the lost dimension in achievement motivation research. En W. Hacker, W. Volpert \& M. von Cranach (Eds.), Cognitive and motivational aspects of action (pp. 8698). Amsterdam: North-Holland.

González, M. L. \& Daura, F. T. (2012). El aprendizaje autorregulado y su vinculación con la perspectiva de futuro. Revista de Orientación Educacional, 26(50), 47-72.

González Lomelí, D., Maytorena, M. A., Lohr Escalante, F. y Carreño Cruz, E. A. (2006). Influencia de la perspectiva temporal y la morosidad académica en estudiantes universitarios. Revista Colombiana de Psicología, (15), 1524.

Herrera, D., Matos, L., Martínez, P. \& Lens, W. (2015). Perspectiva de tiempo futuro y satisfacción con la vida en adolescentes: un estudio transcultural (Dedicado al Prof. Dr. Willy Lens). Revista de Orientación Educacional, 29(55), 37-54.

Husman, J. \& Lens, W. (1999). The Role of the Future in Student Motivation. Educational Psychologist, 34, 113125.

James, W. (1890). The principles of psychology. New York: Holt.

Keough, K. A., Zimbardo, P. G. \& Boyd, J. N. (1999). Who's smoking, drinking and using drugs? Time perspective as a predictor of substance use. Basic and Applied Social Psychology, 21(2), 149-164

Kruger, J. K., Reischl, T. \& Zimmerman, M. A. (2008). Time perspective as mechanism for functional developmental adaptation. Journal of Social, Evolutionary an Cultural Psychology, 2(1), 1-22.

Laplanche, J. (1966). La prioridad del otro en psicoanálisis. Buenos Aires: Amorrortu.

Lens, W. (1998). El rol de la perspectiva de tiempo futuro en la motivación estudiantil. Persona, 1, 67-94.

Lersch, P. H. (1966). Aufbau der person. Munchen: Johann Ambrosius Barth.

Lewin, K. (1935). A dynamic theory of personality. New York: Publisher McGraw - Hill Book.

Lewin, K. (1942). Time perspective and morale. En G. Watson (eds.), Civilian morale. Boston Houghton Mifflin.

Madueño Ruiz, S. (2017). Perspectiva temporal futura en mujeres sentenciadas por el delito de terrorismo en un establecimiento 
penitenciario de Lima (Perú). Investigación y Desarrollo, 25(2), 6181. Doi: 10.14482/indes.25.2.10959

Nurmi, J. (1989). Development of orientation to the future during early adolescence: A four-year longitudinal study and two crosssectional comparisons. International Journal of Psychology, 24(1), 195-214.

Nurmi, J. E. (1991). How do adolescents see their future? A review of the development of future orientation and planning. Developmental Review, 11(1), 1-59.

Nurmi, J.E. (1994). The development of future orientation in life-span context. En Z. Zaleski (Ed.), Psychology of future orientation. Lublin: Towarzystwo Naukowe KUL.

Nuttin, J. (1964). The future time perspective in human motivation and learning. In proceedings of the 17th international Congress of Psychology (pp. 60-82). Amsterdam: NorthHolland.

Nuttin, J. (1985). Future time perspective and Motivation. Louvain: Leuven University Press y Lawrence Erlbaum Associates.

Nuttin J., \& Lens, W. (1985). Future Time Perspective and Motivation: theory and research method. Leuven \& Hillsdale, NJ: Leuven University Press \& Erlbaum.

Roeckelin, J. E. (2000). The concept of time in Psychology. Wesport, Conecticut: Greenwood Press.

San Agustín. (1946). Confesiones. En: Obras de San Agustín, Edición bilingüe, Tomo II, B.A.C., Madrid. Edición crítica
Savio, S. A. (2009). Imágenes de futuro como proyección hacia el futuro: elemento clave para el estudio de la calidad de vida. Revista Académica Hologramática, 1(10), 59-74.

Sircova, A., van de Vijver, F. J. R., Osin, E., Milfont, T. L., Fieulaine, N., Kislali Erginbilgic, A. \& Zimbardo, P. G. (2014). A global look at time: a 24country study of the equivalence of the Zimbardo Time Perspective Inventory. SAGE Open, 4(1), 1-12. Doi: $10.1177 / 2158244013515686$

Sircova, A., van de Vijver, F. J. R., Osin, E., Milfont, T. L., Fieulaine, N., Kislali Erginbilgic, A. \& Zimbardo, P. G. (2015). Time Perspective profiles of cultures. En M. Stolarski, N. Fieulaine \& W. van Beek (Eds.), Time Perspective Theory; Review, Research and Application. Switzerland: SPRINGER. Doi: 10.1007/978-3-319-07368-2

Stolarski, M., Matthews, G., Postek, S., Zimbardo, P. G. \& Bitner, J. (2013). How we feel is a matter of time: relationships between Time Perspectives and mood. Journal of Happiness Studies. Doi: 10.1007/s10902-013-9450-y

Sunddendorf, T. \& Corballis, M.C. (1997). Mental time travel and the evolution of the human mind. Genetic, Social and General Psychology Monographs, 123(2), 133-167.

Thiebaut, E. (1998). La perspective temporelle, un concept a la recherche d'une définition opération nelle. L'Année psychologique, 98(1), 101125. Doi: 10.3406/psy.1998.28614

Vázquez, E. M., Difabio de Anglat, H. E. \& Noriega Biggio, M. (2016). Perspectiva temporal y estilos de 
personalidad en estudiantes argentinos. Interdisciplinaria, 33(2).

Vásquez Echeverría, A. (2011). Experiencia subjetiva del tiempo y su influencia en el comportamiento: Revisión y modelos. Psicologia: Teoria $e$ Pesquisa, 27(2), 215-223.

Webster, J.D. (2011). A new measure of time perspective: Initial psychometric findings for the Balanced Time Perspective Scale (BTPS). Canadian Journal of Behavioural Science, 43 (2), 111-118. Doi: 10.1037/a0022801

Zaleski, Z. (1994). Towards a psychology of the personal future. En Z. Zaleski (Ed.), Psychology of future orientation. Lublin, Polonia: Towarzystwo Naukowe KUL.

Zimbardo, P., Keough, K. \& Boyd, J.N. (1997). Present time perspective as a predictor of risky driving. Personality and Individual Differences, 23(6), 1007-1023.

Doi: https://doi.org/10.1016/S01918869(97)00113-X

Zimbardo, P. G. \& Boyd, J. N. (1999). Putting time in perspective: a valid reliable individual-differences metric. Journal of Personality and Social Psychology, 77(6), 1271-1288.

Zimbardo, P. (2002). Just think about it: Time to take our time. Psychology Today, 35, 62. 